\title{
Semilocal Convergence for a Fifth-Order Newton's Method Using Recurrence Relations in Banach Spaces
}

\author{
Liang Chen, ${ }^{1,2}$ Chuanqing $\mathrm{Gu}^{1}{ }^{1}$ and Yanfang $\mathrm{Ma}^{3}$ \\ ${ }^{1}$ Department of Mathematics, Shanghai University, Shanghai 200444, China \\ 2 School of Mathematical Science, Huaibei Normal University, Anhui, Huaibei 235000, China \\ ${ }^{3}$ School of Computer Science and Technology, Huaibei Normal University, Anhui, Huaibei 235000, China
}

Correspondence should be addressed to Liang Chen, clmyf2@163.com

Received 13 August 2011; Accepted 3 October 2011

Academic Editor: Ke Chen

Copyright (C) 2011 Liang Chen et al. This is an open access article distributed under the Creative Commons Attribution License, which permits unrestricted use, distribution, and reproduction in any medium, provided the original work is properly cited.

We study a modified Newton's method with fifth-order convergence for nonlinear equations in Banach spaces. We make an attempt to establish the semilocal convergence of this method by using recurrence relations. The recurrence relations for the method are derived, and then an existenceuniqueness theorem is given to establish the R-order of the method to be five and a priori error bounds. Finally, a numerical application is presented to demonstrate our approach.

\section{Introduction}

Many scientific problems can be expressed in the form of a nonlinear equation

$$
F(x)=0,
$$

where $F: \Omega \subset X \rightarrow Y$ is a nonlinear operator on an open convex subset $\Omega$ of a Banach space $X$ with values in a Banach space $Y$.

The Newton's method [1, 2], which has quadratically order convergence, is one of the most well-known methods for solving nonlinear equations. Recently, numerous variants of Newton's method with high-order convergence are developed in the literature [3-7]; these methods improve the local order of convergence of Newton's method by an additional evaluation of the function. In this paper, we consider the semilocal convergence for the method proposed in [7]. We first extend this method to Banach spaces and write it as 


$$
\begin{gathered}
u_{n}=x_{n}-\Gamma_{n} F\left(x_{n}\right), \\
z_{n}=x_{n}-\left[F^{\prime}\left(y_{n}\right)\right]^{-1} F\left(x_{n}\right), \\
J_{F}\left(x_{n}, y_{n}\right)=\left[3 F^{\prime}\left(y_{n}\right)-2 F^{\prime}\left(x_{n}\right)\right]^{-1} F^{\prime}\left(y_{n}\right), \\
x_{n+1}=z_{n}-J_{F}\left(x_{n}, y_{n}\right) \Gamma_{n} F\left(z_{n}\right),
\end{gathered}
$$

where $I$ is the identity operator on $X, \Gamma_{n}=\left[F^{\prime}\left(x_{n}\right)\right]^{-1}, y_{n}=x_{n}+(1 / 2)\left(u_{n}-x_{n}\right)$.

In many papers, the convergence of iterative methods for solving nonlinear operator equation in Banach spaces is established from the convergence of majorizing sequences, which is obtained by applying the real function to a polynomial [8-10]. An alternative approach is developed to establish this convergence by using recurrence relations. For example, the recurrence relations are used in establishing the convergence of Newton's method [2] and some high-order methods [11-17]. In this paper, we consider the semilocal convergence of the method given by (1.2) using the recurrence relations. We construct the system of recurrence relations and prove the convergence of the method, along with an error estimate. Finally, numerical results are presented to demonstrate our approach.

\section{Preliminary Results}

Let $x_{0} \in \Omega$, and the nonlinear operator $F: \Omega \subset X \rightarrow Y$ be continuously second-order Fréchet differentiable, where $\Omega$ is an open set and $X$ and $Y$ are Banach spaces. We assume that

(C1) $\left\|\Gamma_{0} F\left(x_{0}\right)\right\| \leq \eta$,

(C2) $\left\|\Gamma_{0}\right\| \leq \beta$,

(C3) $\left\|F^{\prime \prime}(x)\right\| \leq M, x \in \Omega$,

(C4) there exists a positive real number $N$ such that

$$
\left\|F^{\prime \prime}(x)-F^{\prime \prime}(y)\right\| \leq N\|x-y\|, \quad \forall x, y \in \Omega
$$

Now, we define the following scalar functions which will be often used in the later developments. Let

$$
\begin{gathered}
g(t, s)=\frac{2-t}{2-3 t}\left[\frac{t^{3}}{2(2-t)^{2}}+\frac{t^{2}}{4-2 t}+\frac{3}{4} s\right]+\frac{2}{2-t^{\prime}} \\
h(t, s)=\frac{1}{1-\operatorname{tg}(t, s)}, \\
\varphi(t, s)=\frac{t}{2(1-t)^{2}}\left[\frac{t^{3}}{2(2-t)^{2}}+\frac{t^{2}}{4-2 t}+\frac{3}{4} s\right]^{2} \\
+\left(\frac{7 s}{8(1-t)}+\frac{t^{2}}{(2-t)(1-t)}+\frac{2 t^{2}}{2-3 t}\right)\left[\frac{t^{3}}{2(2-t)^{2}}+\frac{t^{2}}{4-2 t}+\frac{3}{4} s\right] .
\end{gathered}
$$


Let $p(t)=\operatorname{tg}(t, s)-1$, and let $\sigma(s)$ be the smallest positive zero of the scalar function $p(t)$ for $s \in(0,5)$. Then, using MATLAB, we obtain that $\sigma(s)$ is decreasing and $\sigma(s)>0$ for all $s \in(0,5)$ and

$$
\max _{0<s<5} \sigma(s) \approx 0.6117085589952558<\frac{2}{3}
$$

Some properties of the functions defined above are given in the following lemma.

Lemma 2.1. Let the real functions $g, h$, and $\varphi$ be given in (2.2). Then

(a) $g(t, s)$ and $h(t, s)$ are increasing and $g(t, s)>1, h(t, s)>1$ for all $t \in(0, \sigma(s))$ and $s \in(0,5)$,

(b) $\varphi(t, s)$ is increasing for all $t \in(0, \sigma(s))$ and $s \in(0,5)$,

(c) $g\left(\theta t, \theta^{2} s\right)<g(t, s), h\left(\theta t, \theta^{2} s\right)<h(t, s)$, and $\varphi\left(\theta t, \theta^{2} s\right)<\theta^{4} \varphi(t, s)$ for $\theta \in(0,1), t \in$ $(0, \sigma(s))$, and $s \in(0,5)$.

Assume that the conditions (C1)-(C4) hold. we now denote $\eta_{0}=\eta, \beta_{0}=\beta, a_{0}=M \beta_{0} \eta_{0}$, $b_{0}=N \beta_{0} \eta_{0}^{2}$, and $c_{0}=h\left(a_{0}, b_{0}\right) \varphi\left(a_{0}, b_{0}\right)$. Let $a_{0}<\sigma(s)$ and $h\left(a_{0}, b_{0}\right) c_{0}<1$, then we can define the following sequences for $n \geq 0$ :

$$
\begin{gathered}
\eta_{n+1}=c_{n} \eta_{n}, \\
\beta_{n+1}=h\left(a_{n}, b_{n}\right) \beta_{n}, \\
a_{n+1}=M \beta_{n+1} \eta_{n+1}, \\
b_{n+1}=N \beta_{n+1} \eta_{n+1}^{2}, \\
c_{n+1}=h\left(a_{n+1}, b_{n+1}\right) \varphi\left(a_{n+1}, b_{n+1}\right) .
\end{gathered}
$$

From the definition of $a_{n+1}, b_{n+1},(2.4)$ and (2.5), we also have

$$
\begin{aligned}
& a_{n+1}=h\left(a_{n}, b_{n}\right) c_{n} a_{n}, \\
& b_{n+1}=h\left(a_{n}, b_{n}\right) c_{n}^{2} b_{n} .
\end{aligned}
$$

Nextly we will study some properties of the previous scalar sequences defined in (2.4)-(2.10), and later developments will require the following lemma.

Lemma 2.2. Let the real functions $g, h$, and $\varphi$ be given in (2.2). If

$$
a_{0}<\sigma(s), \quad h\left(a_{0}, b_{0}\right) c_{0}<1,
$$

then one has

(a) $h\left(a_{0}, b_{0}\right)>1$ and $c_{n}<1$ for $n \geq 0$,

(b) the sequences $\left\{\eta_{n}\right\},\left\{a_{n}\right\},\left\{b_{n}\right\}$, and $\left\{c_{n}\right\}$ are decreasing while $\left\{\beta_{n}\right\}$ is increasing,

(c) $g\left(a_{n}, b_{n}\right) a_{n}<1$ and $h\left(a_{n}, b_{n}\right) c_{n}<1$ for $n \geq 0$. 
Proof. By Lemma 2.1 and (2.11), $h\left(a_{0}, b_{0}\right)>1$ and $c_{n}<1$ hold. It follows from the definitions that $\eta_{1}<\eta_{0}, a_{1}<a_{0}, b_{1}<b_{0}$. Moreover, by Lemma 2.1, we have $1<h\left(a_{1}, b_{1}\right)<h\left(a_{1}, b_{0}\right)<$ $h\left(a_{0}, b_{0}\right)$ and $\varphi\left(a_{1}, b_{1}\right)<\varphi\left(a_{1}, b_{0}\right)<\varphi\left(a_{0}, b_{0}\right)$. This yields $c_{1}<c_{0}, \beta_{1}>\beta_{0}$, and $(\mathrm{b})$ holds. Based on these results we obtain $g\left(a_{1}, b_{1}\right) a_{1}<g\left(a_{0}, b_{0}\right) a_{0}<1$ and $h\left(a_{1}, b_{1}\right) c_{1}<h\left(a_{0}, b_{0}\right) c_{0}<1$ and (c) holds. By induction we can derive that the items (a), (b), and (c) hold.

Lemma 2.3. Under the assumptions of Lemma 2.2 and defining $\gamma=h\left(a_{0}, b_{0}\right) c_{0}$, then

$$
c_{n} \leq \lambda \gamma^{5^{n}}, \quad n \geq 0,
$$

where $\lambda=1 / h\left(a_{0}, b_{0}\right)$. Also, for $n \geq 0$, one has

$$
\prod_{i=0}^{n} c_{i} \leq \lambda^{n+1} \gamma^{\left(5^{n+1}-1\right) / 4}
$$

Proof. By the definition of $a_{n+1}$ and $b_{n+1}$ given in (2.9)-(2.10), we obtain $a_{1}=h\left(a_{0}, b_{0}\right) c_{0} a_{0}=$ $\gamma a_{0}, b_{1}=h\left(a_{0}, b_{0}\right) c_{0}^{2} b_{0}<\gamma^{2} b_{0}$; by Lemma 2.1 we have

$$
c_{1}<h\left(r a_{0}, \gamma^{2} b_{0}\right) \varphi\left(r a_{0}, \gamma^{2} b_{0}\right)<\gamma^{4} h\left(a_{0}, b_{0}\right) \varphi\left(a_{0}, b_{0}\right)=r^{5^{1}-1} c_{0}=\lambda r^{5^{1}}
$$

Suppose $c_{k} \leq \lambda \gamma^{5^{k}}, k \leq 1$. Then, by Lemma 2.2, we have $a_{k+1}<a_{k}, b_{k+1}<b_{k}$ and $h\left(a_{k}, b_{k}\right) c_{k}<$ 1. Thus,

$$
\begin{aligned}
c_{k+1} & <h\left(a_{k}, b_{k}\right) \varphi\left(h\left(a_{k}, b_{k}\right) c_{k} a_{k}, h\left(a_{k}, b_{k}\right) c_{k}^{2} b_{k}\right) \\
& <h\left(a_{k}, b_{k}\right) \varphi\left(h\left(a_{k}, b_{k}\right) c_{k} a_{k}, h^{2}\left(a_{k}, b_{k}\right) c_{k}^{2} b_{k}\right) \\
& <h^{5}\left(a_{k}, b_{k}\right) c_{k}^{4} \varphi\left(a_{k}, b_{k}\right)=h^{4}\left(a_{k}, b_{k}\right) c_{k}^{5} \\
& <\lambda \gamma^{5^{k+1}} .
\end{aligned}
$$

Therefore, it holds that $c_{n} \leq \lambda \gamma^{5^{n}}, n \geq 0$.

By (2.12), we get

$$
\prod_{i=0}^{n} c_{i} \leq \prod_{i=0}^{n} \lambda \gamma^{5^{i}}=\lambda^{n+1} \gamma^{\sum_{i=0}^{n} 5^{i}}=\lambda^{n+1} \gamma^{\left(5^{n+1}-1\right) / 4}, \quad n \geq 0
$$

This shows that (2.13) holds. The proof is completed.

Lemma 2.4. Under the assumptions of Lemma 2.2, let $\gamma=h\left(a_{0}, b_{0}\right) c_{0}$ and $\lambda=1 / h\left(a_{0}, b_{0}\right)$. The sequence $\left\{\eta_{n}\right\}$ satisfies

$$
\eta_{n} \leq \eta \lambda^{n} \gamma^{\left(5^{n}-1\right) / 4}, \quad n \geq 0
$$


Hence, the sequence $\left\{\eta_{n}\right\}$ converges to 0 . Moreover, for any $n \geq 0, m \geq 1$, it holds that

$$
\sum_{i=n}^{n+m} \eta_{i} \leq \eta \lambda^{n} \gamma^{\left(5^{n}-1\right) / 4} \frac{1-\lambda^{m+1} \gamma^{5^{n}\left(5^{m}+3\right) / 4}}{1-\lambda \gamma^{5^{n}}}
$$

Proof. From the definition of sequence $\left\{\eta_{n}\right\}$ given in (2.4) and (2.13), we have

$$
\eta_{n}=c_{n-1} \eta_{n-1}=c_{n-1} c_{n-2} \eta_{n-2}=\cdots=\eta\left(\prod_{i=0}^{n-1} c_{i}\right) \leq \eta \lambda^{n} \gamma^{\left(5^{n}-1\right) / 4}
$$

Because $\lambda<1$ and $\gamma<1$, it follows that $\eta_{n} \rightarrow 0$ as $n \rightarrow \infty$; hence, the sequence $\left\{\eta_{n}\right\}$ converges to 0 .

Since

$$
\begin{aligned}
\sum_{i=n}^{n+m} \lambda^{i} \gamma^{5^{i} / 4} & \leq \lambda^{n} \gamma^{5^{n} / 4}+\gamma^{5^{n}}\left(\sum_{i=n+1}^{n+m} \lambda^{i} \gamma^{5^{i-1} / 4}\right) \\
& =\lambda^{n} \gamma^{5^{n} / 4}+\lambda \gamma^{5^{n}}\left(\sum_{i=n}^{n+m-1} \lambda^{i} \gamma^{5^{i} / 4}\right) \\
& =\lambda^{n} \gamma^{5^{n} / 4}+\lambda \gamma^{5^{n}}\left(\sum_{i=n}^{n+m} \lambda^{i} \gamma^{5^{i} / 4}-\lambda^{n+m} \gamma^{5^{n+m} / 4}\right),
\end{aligned}
$$

where $n \geq 0, m \geq 1$, we can obtain

$$
\sum_{i=n}^{n+m} \lambda^{i} \gamma^{5^{i} / 4} \leq \lambda^{n} \gamma^{5^{n} / 4} \frac{1-\lambda^{m+1} \gamma^{5^{n}\left(5^{m}+3\right) / 4}}{1-\lambda \gamma^{5^{n}}}
$$

Furthermore,

$$
\begin{gathered}
\sum_{i=n}^{n+m} \eta_{i} \leq \eta \sum_{i=n}^{n+m} \lambda^{i} \gamma^{\left(5^{i}-1\right) / 4}=\eta \gamma^{-1 / 4} \sum_{i=n}^{n+m} \lambda^{i} \gamma^{5^{i} / 4} \\
\leq \eta \lambda^{n} \gamma^{\left(5^{n}-1\right) / 4} \frac{1-\lambda^{m+1} \gamma^{5^{n}\left(5^{m}+3\right) / 4}}{1-\lambda \gamma^{5^{n}}}
\end{gathered}
$$

Therefore, $\sum_{n=0}^{\infty} \eta_{n}$ exists. The proof is completed.

\section{Recurrence Relations for the Method}

We firstly give an approximation of the operator $F$ in the following lemma, which will be used in the next derivation. 
Lemma 3.1. Assume that the nonlinear operator $F: \Omega \subset X \rightarrow Y$ is continuously second-order Fréchet differentiable, where $\Omega$ is an open set and $X$ and $Y$ are Banach spaces. Then, one has

$$
\begin{aligned}
F\left(z_{n}\right)= & \int_{0}^{1} F^{\prime \prime}\left(u_{n}+t\left(z_{n}-u_{n}\right)\right)(1-t) d t\left(z_{n}-u_{n}\right)^{2} \\
& -\int_{0}^{1} F^{\prime \prime}\left(y_{n}+t\left(u_{n}-y_{n}\right)\right)\left(u_{n}-y_{n}\right) d t\left[F^{\prime}\left(y_{n}\right)\right]^{-1}\left[F^{\prime}\left(y_{n}\right)-F^{\prime}\left(x_{n}\right)\right]\left(u_{n}-x_{n}\right) \\
& +\int_{0}^{1}\left[F^{\prime \prime}\left(x_{n}+t\left(u_{n}-x_{n}\right)\right)-F^{\prime \prime}\left(x_{n}\right)\right](1-t) d t\left(u_{n}-x_{n}\right)^{2} \\
& +\frac{1}{2} \int_{0}^{1}\left[F^{\prime \prime}\left(x_{n}\right)-F^{\prime \prime}\left(x_{n}+\frac{1}{2} t\left(u_{n}-x_{n}\right)\right)\right] d t\left(u_{n}-x_{n}\right)^{2}, \\
F\left(x_{n+1}\right)= & \int_{0}^{1}\left[F^{\prime \prime}\left(x_{n}+t\left(u_{n}-x_{n}\right)\right)-F^{\prime \prime}\left(x_{n}\right)\right]\left(u_{n}-x_{n}\right) d t\left(x_{n+1}-z_{n}\right) \\
& -\frac{3}{2} \int_{0}^{1}\left[F^{\prime \prime}\left(x_{n}+\frac{1}{2} t\left(u_{n}-x_{n}\right)\right)-F^{\prime \prime}\left(x_{n}\right)\right]\left(u_{n}-x_{n}\right) d t\left(x_{n+1}-z_{n}\right) \\
& -2 F^{\prime \prime}\left(x_{n}\right)\left(y_{n}-x_{n}\right)\left[I+3 H\left(x_{n}, y_{n}\right)\right]^{-1} H\left(x_{n}, y_{n}\right) \Gamma_{n} F\left(z_{n}\right) \\
& -\frac{1}{2} \int_{0}^{1}\left[F^{\prime \prime}\left(x_{n}+\frac{1}{2} t\left(u_{n}-x_{n}\right)\right)-F^{\prime \prime}\left(x_{n}\right)\right]\left(u_{n}-x_{n}\right) d t \Gamma_{n} F\left(z_{n}\right) \\
& +\int_{0}^{1}\left[F^{\prime}\left(z_{n}+t\left(x_{n+1}-z_{n}\right)\right)-F^{\prime}\left(u_{n}\right)\right] d t\left(x_{n+1}-z_{n}\right),
\end{aligned}
$$

where $H\left(x_{n}, y_{n}\right)=\Gamma_{n}\left[F^{\prime}\left(y_{n}\right)-F^{\prime}\left(x_{n}\right)\right]$ and $J_{F}\left(x_{n}, y_{n}\right)=I-2\left[I+3 H\left(x_{n}, y_{n}\right)\right]^{-1} H\left(x_{n}, y_{n}\right)$.

Proof. By the Taylor Expansion, we obtain

$$
\begin{aligned}
F\left(z_{n}\right)= & F\left(u_{n}\right)+F^{\prime}\left(u_{n}\right)\left(z_{n}-u_{n}\right)+\int_{0}^{1} F^{\prime \prime}\left(u_{n}+t\left(z_{n}-u_{n}\right)\right)(1-t) d t\left(z_{n}-u_{n}\right)^{2} \\
F\left(u_{n}\right)= & F\left(x_{n}\right)+F^{\prime}\left(x_{n}\right)\left(u_{n}-x_{n}\right)+\frac{1}{2} F^{\prime \prime}\left(x_{n}\right)\left(u_{n}-x_{n}\right)^{2} \\
& +\int_{0}^{1}\left[F^{\prime \prime}\left(x_{n}+t\left(u_{n}-x_{n}\right)\right)-F^{\prime \prime}\left(x_{n}\right)\right](1-t) d t\left(u_{n}-x_{n}\right)^{2} \\
F^{\prime}\left(u_{n}\right)= & F^{\prime}\left(y_{n}\right)+\int_{0}^{1} F^{\prime \prime}\left(y_{n}+t\left(u_{n}-y_{n}\right)\right)\left(u_{n}-y_{n}\right) d t \\
F^{\prime}\left(y_{n}\right)= & F^{\prime}\left(x_{n}\right)+\frac{1}{2} F^{\prime \prime}\left(x_{n}\right)\left(u_{n}-x_{n}\right)+\frac{1}{2} \int_{0}^{1}\left[F^{\prime \prime}\left(x_{n}+\frac{1}{2} t\left(u_{n}-x_{n}\right)\right)-F^{\prime \prime}\left(x_{n}\right)\right] d t\left(u_{n}-x_{n}\right)
\end{aligned}
$$


and we obtain

$$
\begin{aligned}
F^{\prime}\left(u_{n}\right)\left(z_{n}-u_{n}\right)= & F^{\prime}\left(y_{n}\right)\left(z_{n}-u_{n}\right)+\int_{0}^{1} F^{\prime \prime}\left(y_{n}+t\left(u_{n}-y_{n}\right)\right)\left(u_{n}-y_{n}\right) d t\left(z_{n}-u_{n}\right), \\
{\left[F^{\prime}\left(y_{n}\right)-F^{\prime}\left(x_{n}\right)\right]\left(u_{n}-x_{n}\right)=} & \frac{1}{2} F^{\prime \prime}\left(x_{n}\right)\left(u_{n}-x_{n}\right)^{2} \\
& +\frac{1}{2} \int_{0}^{1}\left[F^{\prime \prime}\left(x_{n}+\frac{1}{2} t\left(u_{n}-x_{n}\right)\right)-F^{\prime \prime}\left(x_{n}\right)\right] d t\left(u_{n}-x_{n}\right)^{2} .
\end{aligned}
$$

By the first two steps of method given in (1.2) and (3.4), (3.6), (3.7), we obtain

$$
\begin{aligned}
F^{\prime}\left(u_{n}\right)\left(z_{n}-u_{n}\right)= & -\frac{1}{2} F^{\prime \prime}\left(x_{n}\right)\left(u_{n}-x_{n}\right)^{2} \\
& +\frac{1}{2} \int_{0}^{1}\left[F^{\prime \prime}\left(x_{n}\right)-F^{\prime \prime}\left(x_{n}+\frac{1}{2} t\left(u_{n}-x_{n}\right)\right)\right] d t\left(u_{n}-x_{n}\right)^{2} \\
& +\int_{0}^{1} F^{\prime \prime}\left(y_{n}+t\left(u_{n}-y_{n}\right)\right)\left(u_{n}-y_{n}\right) d t\left(z_{n}-u_{n}\right) .
\end{aligned}
$$

Substituting (3.4) and (3.8) into (3.3), we obtain (3.1).

We now consider $F\left(x_{n+1}\right)$. Since

$$
\left[3 F^{\prime}\left(y_{n}\right)-2 F^{\prime}\left(x_{n}\right)\right]\left(x_{n+1}-z_{n}\right)+\left[F^{\prime}\left(y_{n}\right)-F^{\prime}\left(x_{n}\right)\right] \Gamma_{n} F\left(z_{n}\right)+F\left(z_{n}\right)=0,
$$

using Taylor's formula, we have

$$
\begin{aligned}
F\left(x_{n+1}\right)= & F\left(z_{n}\right)+F^{\prime}\left(u_{n}\right)\left(x_{n+1}-z_{n}\right)+\int_{0}^{1}\left[F^{\prime}\left(z_{n}+t\left(x_{n+1}-z_{n}\right)\right)-F^{\prime}\left(u_{n}\right)\right] d t\left(x_{n+1}-z_{n}\right) \\
= & {\left[F^{\prime}\left(u_{n}\right)-F^{\prime}\left(y_{n}\right)\right]\left(x_{n+1}-z_{n}\right)-2\left[F^{\prime}\left(y_{n}\right)-F^{\prime}\left(x_{n}\right)\right]\left(x_{n+1}-z_{n}\right) } \\
& -\left[F^{\prime}\left(y_{n}\right)-F^{\prime}\left(x_{n}\right)\right] \Gamma_{n} F\left(z_{n}\right)+\int_{0}^{1}\left[F^{\prime}\left(z_{n}+t\left(x_{n+1}-z_{n}\right)\right)-F^{\prime}\left(u_{n}\right)\right] d t\left(x_{n+1}-z_{n}\right) .
\end{aligned}
$$

Similarly, we obtain

$$
F^{\prime}\left(u_{n}\right)=F^{\prime}\left(x_{n}\right)+F^{\prime \prime}\left(x_{n}\right)\left(u_{n}-x_{n}\right)+\int_{0}^{1}\left[F^{\prime \prime}\left(x_{n}+t\left(u_{n}-x_{n}\right)\right)-F^{\prime \prime}\left(x_{n}\right)\right]\left(u_{n}-x_{n}\right) d t
$$


It follows that

$$
\begin{aligned}
& F^{\prime}\left(u_{n}\right)-F^{\prime}\left(y_{n}\right)=\frac{1}{2} F^{\prime \prime}\left(x_{n}\right)\left(u_{n}-x_{n}\right)+\int_{0}^{1}\left[F^{\prime \prime}\left(x_{n}+t\left(u_{n}-x_{n}\right)\right)-F^{\prime \prime}\left(x_{n}\right)\right]\left(u_{n}-x_{n}\right) d t \\
& \quad-\frac{1}{2} \int_{0}^{1}\left[F^{\prime \prime}\left(x_{n}+\frac{1}{2} t\left(u_{n}-x_{n}\right)\right)-F^{\prime \prime}\left(x_{n}\right)\right]\left(u_{n}-x_{n}\right) d t \\
& F^{\prime}\left(y_{n}\right)-F^{\prime}\left(x_{n}\right)=\frac{1}{2} F^{\prime \prime}\left(x_{n}\right)\left(u_{n}-x_{n}\right)+\frac{1}{2} \int_{0}^{1}\left[F^{\prime \prime}\left(x_{n}+\frac{1}{2} t\left(u_{n}-x_{n}\right)\right)-F^{\prime \prime}\left(x_{n}\right)\right]\left(u_{n}-x_{n}\right) d t .
\end{aligned}
$$

Substituting (3.12) into (3.10), we can obtain (3.2). The proof is completed.

We denote $B(x, r)=\{y \in X:\|y-x\|<r\}$ and $\overline{B(x, r)}=\{y \in X:\|y-x\| \leq r\}$ in this paper.

In the following, the recurrence relations are derived for the method given by (1.2) under the assumptions mentioned in the previous section.

For $n=0$, the existence of $\Gamma_{0}$ implies the existence of $u_{0}, y_{0}$. This gives us

$$
\left\|u_{0}-x_{0}\right\|=\left\|\Gamma_{0} F\left(x_{0}\right)\right\| \leq \eta_{0}
$$

and this means that $u_{0}, y_{0} \in B\left(x_{0}, R \eta\right)$, where $R=g\left(a_{0}, b_{0}\right) /\left(1-c_{0}\right)$. By the initial hypotheses, we have

$$
\left\|H\left(x_{0}, y_{0}\right)\right\| \leq\left\|\Gamma_{0}\right\|\left\|F^{\prime}\left(x_{0}\right)-F^{\prime}\left(y_{0}\right)\right\| \leq \frac{1}{2} M\left\|\Gamma_{0}\right\|\left\|u_{0}-x_{0}\right\| \leq \frac{1}{2} a_{0} .
$$

Because of the assumption $a_{0}<\sigma(s)<2 / 3$, by the Banach lemma [2] it follows that

$$
\left\|\left[I+3 H\left(x_{0}, y_{0}\right)\right]^{-1}\right\| \leq \frac{1}{1-(3 / 2) a_{0}} .
$$

It is followed that

$$
\left\|J_{F}\left(x_{0}, y_{0}\right)\right\| \leq 1+2\left\|\left[I+3 H\left(x_{0}, y_{0}\right)\right]^{-1}\right\|\left\|H\left(x_{0}, y_{0}\right)\right\| \leq 1+\frac{2 a_{0}}{2-3 a_{0}} \text {. }
$$

Consequently $z_{0}$ is well defined and

$$
\left\|z_{0}-x_{0}\right\| \leq\left\|\left[F^{\prime}\left(y_{0}\right)\right]^{-1} F\left(x_{0}\right)\right\| \leq\left\|\left[F^{\prime}\left(y_{0}\right)\right]^{-1} F^{\prime}\left(x_{0}\right)\right\|\left\|\Gamma_{0} F\left(x_{0}\right)\right\| \leq \frac{2}{2-a_{0}} \eta_{0} .
$$


It is similar to obtain

$$
\begin{aligned}
\left\|z_{0}-u_{0}\right\| & =\left\|\left[F^{\prime}\left(y_{0}\right)^{-1}-\Gamma_{0}\right] F\left(x_{0}\right)\right\| \\
& =\left\|F^{\prime}\left(y_{0}\right)^{-1}\left[F^{\prime}\left(x_{0}\right)-F^{\prime}\left(y_{0}\right)\right] \Gamma_{0} F\left(x_{0}\right)\right\| \\
& \leq\left\|F^{\prime}\left(y_{0}\right)^{-1}\right\|\left\|\left[F^{\prime}\left(x_{0}\right)-F^{\prime}\left(y_{0}\right)\right]\right\|\left\|\Gamma_{0} F\left(x_{0}\right)\right\| \\
& \leq \frac{a_{0}}{2-a_{0}} \eta_{0} .
\end{aligned}
$$

By Lemma 3.1, we can get

$$
\begin{gathered}
\left\|F\left(z_{0}\right)\right\| \leq \frac{1}{2} M\left\|z_{0}-u_{0}\right\|^{2}+M^{2}\left\|F^{\prime}\left(y_{0}\right)^{-1}\right\|\left\|u_{0}-y_{0}\right\|\left\|y_{0}-x_{0}\right\|\left\|u_{0}-x_{0}\right\|+\frac{3}{4} N\left\|u_{0}-x_{0}\right\|^{3} \\
\left\|F\left(x_{1}\right)\right\| \leq \frac{1}{2} M\left\|x_{1}-z_{0}\right\|^{2}+\left(M\left\|z_{0}-u_{0}\right\|+\frac{3}{2} N\left\|u_{0}-x_{0}\right\|^{2}\right)\left\|x_{1}-z_{0}\right\| .
\end{gathered}
$$

Therefore, we have

$$
\begin{gathered}
\left\|x_{1}-z_{0}\right\| \leq\left\|J_{F}\left(x_{0}, y_{0}\right)\right\|\left\|\Gamma_{0}\right\|\left\|F\left(z_{0}\right)\right\| \leq \frac{2-a_{0}}{2-3 a_{0}}\left[\frac{a_{0}}{2}\left(\frac{a_{0}}{2-a_{0}}\right)^{2}+\frac{a_{0}^{2}}{2\left(2-a_{0}\right)}+\frac{3}{4} b_{0}\right] \eta_{0}, \\
\left\|x_{1}-x_{0}\right\| \leq\left\|x_{1}-z_{0}\right\|+\left\|z_{0}-x_{0}\right\| \leq g\left(a_{0}, b_{0}\right) \eta_{0} .
\end{gathered}
$$

From the assumption $c_{0}<1 / h\left(a_{0}, b_{0}\right)<1$, it follows that $x_{1} \in B\left(x_{0}, R \eta\right)$.

By $a_{0}<\sigma(s)$ and $g(t, s)$ is increasing in $t \in(0, \sigma(s))$ and $s \in(0,5)$, we have

$$
\begin{aligned}
\left\|I-\Gamma_{0} F^{\prime}\left(x_{1}\right)\right\| & \leq\left\|\Gamma_{0}\right\|\left\|F^{\prime}\left(x_{0}\right)-F^{\prime}\left(x_{1}\right)\right\| \\
& \leq M \beta_{0}\left\|x_{1}-x_{0}\right\| \leq a_{0} g\left(a_{0}, b_{0}\right)<1,
\end{aligned}
$$

and it follows by the Banach lemma [2] that $\Gamma_{1}=\left[F^{\prime}\left(x_{1}\right)\right]^{-1}$ exists and

$$
\left\|\Gamma_{1}\right\| \leq \frac{\beta_{0}}{1-a_{0} g\left(a_{0}, b_{0}\right)}=h\left(a_{0}, b_{0}\right) \beta_{0}=\beta_{1} .
$$

Then, from (3.20) and (3.24), we have

$$
\begin{aligned}
\left\|u_{1}-x_{1}\right\| & =\left\|\Gamma_{1} F\left(x_{1}\right)\right\| \leq\left\|\Gamma_{1}\right\|\left\|F\left(x_{1}\right)\right\| \\
& \leq h\left(a_{0}, b_{0}\right) \varphi\left(a_{0}, b_{0}\right) \eta_{0}=c_{0} \eta_{0}=\eta_{1} .
\end{aligned}
$$


Because of $g\left(a_{0}, b_{0}\right)>1$, we obtain

$$
\begin{aligned}
\left\|u_{1}-x_{0}\right\| & \leq\left\|u_{1}-x_{1}\right\|+\left\|x_{1}-x_{0}\right\| \\
& \leq\left(g\left(a_{0}, b_{0}\right)+c_{0}\right) \eta_{0} \\
& <g\left(a_{0}, b_{0}\right)\left(1+c_{0}\right) \eta<R \eta,
\end{aligned}
$$

which shows $u_{1}, y_{1} \in B\left(x_{0}, R \eta\right)$.

In addition, we have

$$
\begin{aligned}
& M\left\|\Gamma_{1}\right\|\left\|\Gamma_{1} F\left(x_{1}\right)\right\| \leq h\left(a_{0}, b_{0}\right) c_{0} a_{0}=a_{1}, \\
& N\left\|\Gamma_{1}\right\|\left\|\Gamma_{1} F\left(x_{1}\right)\right\|^{2} \leq h\left(a_{0}, b_{0}\right) c_{0}^{2} b_{0}=b_{1} .
\end{aligned}
$$

Repeating the above derivation, we can obtain the system of recurrence relations given in the next lemma.

Lemma 3.2. Let the assumptions and the conditions (C1)-(C4) hold. Then, the following items are true for all $n \geq 0$ :

(i) there exists $\Gamma_{n}=\left[F^{\prime}\left(x_{n}\right)\right]^{-1}$ and $\left\|\Gamma_{n}\right\| \leq \beta_{n}$,

(ii) $\left\|\Gamma_{n} F\left(x_{n}\right)\right\| \leq \eta_{n}$

(iii) $M\left\|\Gamma_{n}\right\|\left\|\Gamma_{n} F\left(x_{n}\right)\right\| \leq a_{n}$,

(iv) $N\left\|\Gamma_{n}\right\|\left\|\Gamma_{n} F\left(x_{n}\right)\right\|^{2} \leq b_{n}$,

(v) $\left\|x_{n+1}-x_{n}\right\| \leq g\left(a_{n}, b_{n}\right) \eta_{n}$

(vi) $\left\|x_{n+1}-x_{0}\right\| \leq R \eta$, where $R=g\left(a_{0}, b_{0}\right) /\left(1-c_{0}\right)$.

Proof. The proof of $(\mathrm{I})-(\mathrm{V})$ follows by using the above-mentioned way and invoking the induction hypothesis. We only consider (VI). By (V) and Lemma 2.4, we obtain

$$
\begin{aligned}
\left\|x_{n+1}-x_{0}\right\| & \leq \sum_{i=0}^{n}\left\|x_{i+1}-x_{i}\right\| \\
& \leq \sum_{i=0}^{n} g\left(a_{i}, b_{i}\right) \eta_{i} \\
& \leq g\left(a_{0}, b_{0}\right) \sum_{i=0}^{n} \eta_{i} \\
& \leq g\left(a_{0}, b_{0}\right) \eta \frac{1-\lambda^{n+1} \gamma^{\left(5^{n}+3\right) / 4}}{1-c_{0}}<R \eta .
\end{aligned}
$$

So the lemma is proved. 


\section{Semilocal Convergence}

Lemma 4.1. Let $R=g\left(a_{0}, b_{0}\right) /\left(1-c_{0}\right)$. If $h\left(a_{0}, b_{0}\right) c_{0}<1$, then $R<1 / a_{0}$.

Proof. Since

$$
c_{0}<\frac{1}{h\left(a_{0}, b_{0}\right)}=1-a_{0} g\left(a_{0}, b_{0}\right)
$$

we obtain $R<1 / a_{0}$.

Now we give a theorem to establish the semilocal convergence of (1.2), the existence and uniqueness of the solution, and the domain in which it is located, along with a priori error bounds, which lead to the $R$-order of convergence at least five of iteration (1.2).

Theorem 4.2. Let $F: \Omega \subset X \rightarrow Y$ be a nonlinear two times Fréchet differentiable operator in an open convex subset $\Omega$ of a Banach space $X$ with values in a Banach space $Y$. Assume that $x_{0} \in \Omega$ and all conditions (C1)-(C4) hold. Let $a_{0}=M \beta \eta, b_{0}=N \beta \eta^{2}$, and $c_{0}=h\left(a_{0}, b_{0}\right) \varphi\left(a_{0}, b_{0}\right)$ satisfy $a_{0}<\sigma(s)$ and $h\left(a_{0}, b_{0}\right) c_{0}<1$, where $g$, $h$, and $\varphi$ are defined by (2.2). Let $\overline{B\left(x_{0}, R \eta\right)} \subset \Omega$, where $R=g\left(a_{0}, b_{0}\right) /\left(1-c_{0}\right)$, then, starting from $x_{0}$, the sequence $\left\{x_{n}\right\}$ generated by the method (1.2) converges to a solution $x^{*}$ of $F(x)$ with $x_{n}, x^{*}$ belonging to $\overline{B\left(x_{0}, R \eta\right)}$ and $x^{*}$ being the unique solution of $F(x)$ in $B\left(x_{0},(2 / M \beta)-R \eta\right) \cap \Omega$. Moreover, a priori error estimate is given by

$$
\left\|x_{n}-x^{*}\right\| \leq \frac{g\left(a_{0}, b_{0}\right) \eta \lambda^{n} \gamma^{\left(5^{n}-1\right) / 4}}{1-\lambda \gamma^{5^{n}}}
$$

where $\gamma=h\left(a_{0}, b_{0}\right) c_{0}$ and $\lambda=1 / h\left(a_{0}, b_{0}\right)$.

Proof. By Lemma 3.2, the sequence $\left\{x_{n}\right\}$ is well defined in $\overline{B\left(x_{0}, R \eta\right)}$. Now we prove that $\left\{x_{n}\right\}$ is a Cauchy sequence. Since

$$
\begin{aligned}
\left\|x_{n+m}-x_{n}\right\| & \leq \sum_{i=n}^{n+m-1}\left\|x_{i+1}-x_{i}\right\| \\
& \leq \sum_{i=n}^{n+m-1} g\left(a_{i}, b_{i}\right) \eta_{i} \\
& \leq g\left(a_{0}, b_{0}\right) \sum_{i=n}^{n+m-1} \eta_{i} \\
& \leq g\left(a_{0}, b_{0}\right) \eta \lambda^{n} \gamma^{\left(5^{n}-1\right) / 4} \frac{1-\lambda^{m} \gamma^{5^{n}\left(5^{m-1}+3\right) / 4}}{1-\lambda \gamma^{5^{n}}},
\end{aligned}
$$

it follows that $\left\{x_{n}\right\}$ is a Cauchy sequence, and hence the sequence $\left\{x_{n}\right\}$ is convergent. So there exists an $x^{*}$ such that $\lim _{n \rightarrow \infty} x_{n}=x^{*}$. 
By letting $n=0, m \rightarrow \infty$ in (4.3), we obtain

$$
\left\|x^{*}-x_{0}\right\| \leq R \eta
$$

This shows that $x^{*} \in \overline{B\left(x_{0}, R \eta\right)}$.

Now we prove that $x^{*}$ is a solution of $F(x)=0$. Since

$$
\left\|\Gamma_{0}\right\|\left\|F\left(x_{n}\right)\right\| \leq\left\|\Gamma_{n}\right\|\left\|F\left(x_{n}\right)\right\| \leq \eta_{n}
$$

by letting $n \rightarrow \infty$ in (4.5), we obtain $\left\|F\left(x_{n}\right)\right\| \rightarrow 0$ since $g\left(a_{n}, b_{n}\right)<g\left(a_{0}, b_{0}\right)$ and $\eta_{n} \rightarrow 0$. Hence, by the continuity of $F$ in $\Omega$, we obtain $F\left(x^{*}\right)=0$.

We prove the uniqueness of $x^{*}$ in $B\left(x_{0},(2 / M \beta)-R \eta\right) \cap \Omega$. Firstly we can obtain $x^{*} \in$ $B\left(x_{0},(2 / M \beta)-R \eta\right) \cap \Omega$, since it follows by Lemma 3.2 that

$$
\frac{2}{M \beta}-R \eta=\left(\frac{2}{a_{0}}-R\right) \eta>\frac{1}{a_{0}} \eta>R \eta
$$

and then $\overline{B\left(x_{0}, R \eta\right)} \subset B\left(x_{0},(2 / M \beta)-R \eta\right) \cap \Omega$. Let $x^{* *}$ be another zero of $F(x)$ in $B\left(x_{0},(2 / M \beta)-\right.$ $R \eta) \cap \Omega$. By Taylor theorem, we have

$$
0=F\left(x^{* *}\right)-F\left(x^{*}\right)=\int_{0}^{1} F^{\prime}\left((1-t) x^{*}+t x^{* *}\right) d t\left(x^{* *}-x^{*}\right)
$$

Since

$$
\begin{aligned}
\left\|\Gamma_{0}\right\|\left\|\int_{0}^{1}\left[F^{\prime}\left((1-t) x^{*}+t x^{* *}\right)-F^{\prime}\left(x_{0}\right)\right] d t\right\| & \leq M \beta \int_{0}^{1}\left[(1-t)\left\|x^{*}-x_{0}\right\|+t\left\|x^{* *}-x_{0}\right\|\right] d t \\
& <\frac{M \beta}{2}\left[R \eta+\frac{2}{M \beta}-R \eta\right]=1,
\end{aligned}
$$

it follows by the Banach lemma that $\int_{0}^{1} F^{\prime}\left((1-t) x^{*}+t x^{* *}\right) d t$ is invertible and hence $x^{* *}=x^{*}$. Finally, by letting $m \rightarrow \infty$ in (4.3), we obtain (4.2) and furthermore

$$
\left\|x_{n}-x^{*}\right\| \leq \frac{g\left(a_{0}, b_{0}\right) \eta}{r^{1 / 4}\left(1-c_{0}\right)}\left(r^{1 / 4}\right)^{5^{n}}
$$

This means that the method given by (1.2) is of $R$-order of convergence at least five. This ends the proof. 


\section{Numerical Example}

Let $X=C[0,1]$ be the space of continuous functions defined on the interval $[0,1]$, with the max-norm, and consider the integral equation $F(x)=0$, where

$$
F(x)(s)=x(s)-1-\frac{1}{2} \int_{0}^{1} s \cos (x(t)) d t
$$

with $s \in[0,1], x \in \Omega=B(0,2) \subset X$. Integral equations of this kind (called Chandrasekhar equations) arise in elasticity or neutron transport problems.

It is easy to obtain the derivatives of $F$ as

$$
\begin{aligned}
& F^{\prime}(x) y(s)=y(s)-\frac{1}{2} \int_{0}^{1} s \sin (x(t)) y(t) d t, \quad y \in \Omega, \\
& F^{\prime \prime}(x) y z(s)=-\frac{1}{2} \int_{0}^{1} s \cos (x(t)) y(t) z(t) d t, \quad y, z \in \Omega,
\end{aligned}
$$

The derivative $F^{\prime \prime}$ satisfies

$$
\left\|F^{\prime \prime}(x)\right\| \leq \frac{1}{2}=M, \quad x \in \Omega,
$$

and the Lipschitz condition with $N=1 / 2$

$$
\left\|F^{\prime \prime}(x)-F^{\prime \prime}(y)\right\| \leq \frac{1}{2}\|x-y\|, \quad x, y \in \Omega,
$$

since the norm is taken as max-norm.

Starting at $x_{0}(t)=4 / 3$, we have

$$
\left\|F\left(x_{0}\right)\right\|=\left\|\frac{1}{2} \int_{0}^{1} s \cos \left(x_{0}(t)\right) d t\right\| \leq \frac{1}{2} \cos \frac{4}{3},
$$

since

$$
\left\|I-F^{\prime}\left(x_{0}\right)\right\|=\left\|\frac{1}{2} \int_{0}^{1} s \sin \left(x_{0}(t)\right) d t\right\| \leq \frac{1}{2} \sin \frac{4}{3}
$$

and by the Banach lemma that $\Gamma_{0}$ exists and

$$
\left\|\Gamma_{0}\right\| \leq \frac{2}{2-\sin (4 / 3)}=\beta
$$


Table 1: Results of recurrence relations.

\begin{tabular}{cccccc}
\hline$n$ & $\eta_{n}$ & $\beta_{n}$ & $a_{n}$ & $b_{n}$ & $c_{n}$ \\
\hline 0 & $2.2882 e-001$ & 1.9454 & $2.2257 e-001$ & $5.0928 e-002$ & $1.3343 e-002$ \\
1 & $3.0530 e-003$ & 2.6520 & $4.0483 e-003$ & $1.2360 e-005$ & $4.7865 e-010$ \\
2 & $1.4613 e-012$ & 2.6628 & $1.9456 e-012$ & $2.8432 e-024$ & $2.5141 e-047$ \\
3 & $3.6740 e-059$ & 2.6628 & $4.8916 e-059$ & $1.7972 e-117$ & $1.0045 e-233$ \\
4 & $3.6904 e-292$ & 2.6628 & $4.9134 e-292$ & 0 & 0 \\
\hline
\end{tabular}

It follow that

$$
\left\|\Gamma_{0} F\left(x_{0}\right)\right\| \leq \frac{\cos (4 / 3)}{2-\sin (4 / 3)}=\eta
$$

Consequently, we obtain

$$
\begin{gathered}
a_{0}=M \beta \eta=\frac{\cos (4 / 3)}{(2-\sin (4 / 3))^{2}}, \\
b_{0}=N \beta \eta^{2}=\frac{\cos ^{2}(4 / 3)}{(2-\sin (4 / 3))^{3}}, \\
c_{0}=h\left(a_{0}, b_{0}\right) \varphi\left(a_{0}, b_{0}\right),
\end{gathered}
$$

which satisfy

$$
\begin{gathered}
a_{0}=0.22257070108520<\max _{b_{n}} \sigma\left(b_{n}\right) \approx 0.6034780649041629<\frac{2}{3}, \\
\mathcal{C}_{0} h\left(a_{0}, b_{0}\right)=0.01818885859745<1 .
\end{gathered}
$$

This means that the hypothesis of Theorem 4.2 is satisfied. Hence, the recurrence relations for the method given by (1.2) are demonstrated in Table 1. Besides, the solution $x^{*}$ belongs to $\overline{B\left(x_{0}, R \eta\right)}=\overline{B(4 / 3,0.27761986977716)} \subset \Omega$, and it is unique in $B(4 / 3,1.77850432749622) \cap \Omega$.

\section{Conclusions}

A family of recurrence relations is developed for establishing the semilocal convergence of a modified Newton's method (1.2) used for solving $F(x)=0$ in Banach spaces. Based on these recurrence relations, an existence uniqueness theorem is established to show the $R$ order convergence of the method to be five. Also a priori error bounds are given. A numerical example is worked out to demonstrate our approach and show that our method can be of practical interest. 


\section{Acknowledgments}

The work is supported by the Shanghai Natural Science Foundation (no. 10ZR1410900), the Key Disciplines of Shanghai Municipality (no. S30104), the Natural Science Research Funds of Anhui Provincial for Universities (Grant no. KJ2011A248), and the Open Fund of Shanghai Key Laboratory of Trustworthy Computing.

\section{References}

[1] J. M. Ortega and W. C. Rheinboldt, Iterative Solution of Nonlinear Equations in Several Variables, Academic Press, New York, NY, USA, 1970.

[2] L. B. Rall, Computational Solution of Nonlinear Operator Equations, John Wiley \& Sons Inc., New York, 1969.

[3] A. Y. Özban, "Some new variants of Newton's method," Applied Mathematics Letters, vol. 17, no. 6, pp. 677-682, 2004.

[4] M. Frontini and E. Sormani, "Some variant of Newton's method with third-order convergence," Applied Mathematics and Computation, vol. 140, no. 2-3, pp. 419-426, 2003.

[5] M. A. Noor and K. I. Noor, "Modified iterative methods with cubic convergence for solving nonlinear equations," Applied Mathematics and Computation, vol. 184, no. 2, pp. 322-325, 2007.

[6] J. Kou and Y. Li, "The improvements of Chebyshev-Halley methods with fifth-order convergence," Applied Mathematics and Computation, vol. 188, no. 1, pp. 143-147, 2007.

[7] J. Kou, "The improvements of modified Newton's method," Applied Mathematics and Computation, vol. 189, no. 1, pp. 602-609, 2007.

[8] F. Cianciaruso and E. de Pascale, "Estimates of majorizing sequences in the Newton-Kantorovich method: a further improvement," Journal of Mathematical Analysis and Applications, vol. 322, no. 1, pp. 329-335, 2006.

[9] J. Chen, I. K. Argyros, and R. P. Agarwal, "Majorizing functions and two-point Newton-type methods," Journal of Computational and Applied Mathematics, vol. 234, no. 5, pp. 1473-1484, 2010.

[10] I. K. Argyros, Y. J. Cho, and S. Hilout, "On the midpoint method for solving equations," Applied Mathematics and Computation, vol. 216, no. 8, pp. 2321-2332, 2010.

[11] C. Chun, P. Stănică, and B. Neta, "Third-order family of methods in Banach spaces," Computers $\mathcal{E}$ Mathematics with Applications, vol. 61, no. 6, pp. 1665-1675, 2011.

[12] J. A. Ezquerro, M. A. Hernández, and M. A. Salanova, "Recurrence relations for the midpoint method," Tamkang Journal of Mathematics, vol. 31, no. 1, pp. 33-41, 2000.

[13] M. A. Hernández, "Chebyshev's approximation algorithms and applications," Computers \& Mathematics with Applications, vol. 41, no. 3-4, pp. 433-445, 2001.

[14] X. Ye and C. Li, "Convergence of the family of the deformed Euler-Halley iterations under the Hölder condition of the second derivative," Journal of Computational and Applied Mathematics, vol. 194, no. 2, pp. 294-308, 2006.

[15] P. K. Parida and D. K. Gupta, "Recurrence relations for a Newton-like method in Banach spaces," Journal of Computational and Applied Mathematics, vol. 206, no. 2, pp. 873-887, 2007.

[16] X. Wang, C. Gu, and J. Kou, "Semilocal convergence of a multipoint fourth-order super-Halley method in Banach spaces," Numerical Algorithms, vol. 56, no. 4, pp. 497-516, 2011.

[17] I. K. Argyros, J. A. Ezquerro, J. M. Gutiérrez, M. A. Hernández, and S. Hilout, "On the semilocal convergence of efficient Chebyshev-Secant-type methods," Journal of Computational and Applied Mathematics, vol. 235, no. 10, pp. 3195-3206, 2011. 


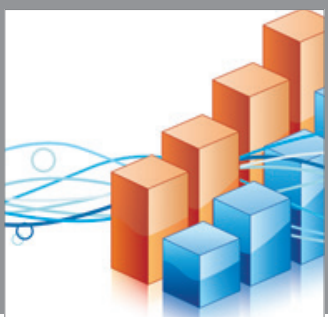

Advances in

Operations Research

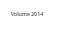

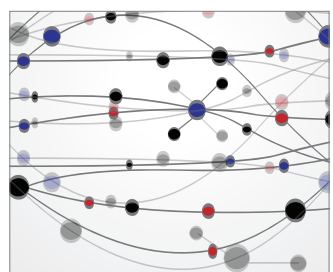

\section{The Scientific} World Journal
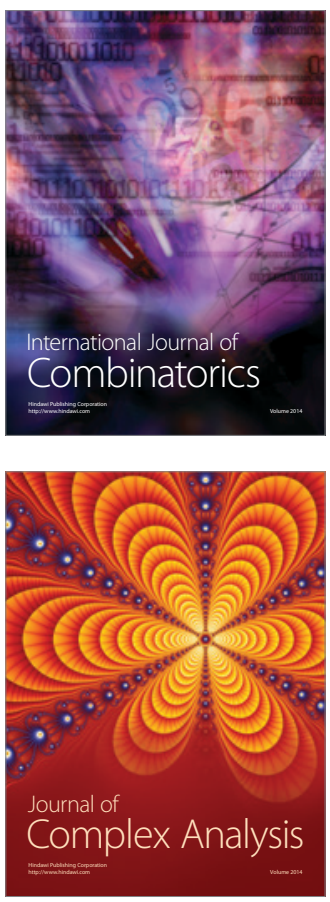

International Journal of

Mathematics and

Mathematical

Sciences
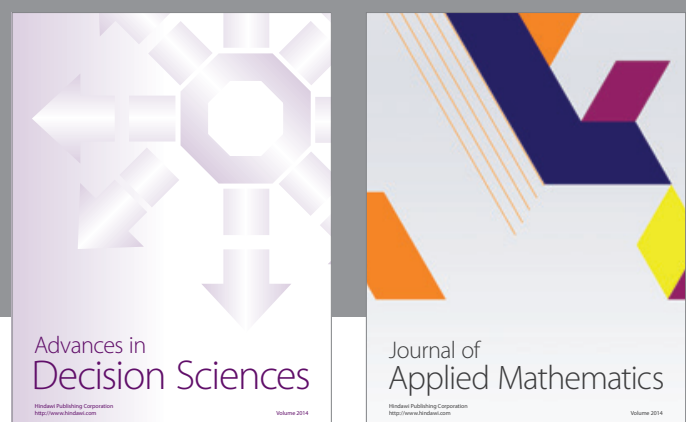

Journal of

Applied Mathematics
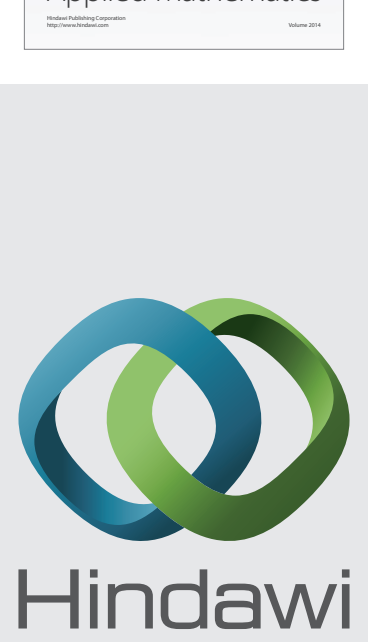

Submit your manuscripts at http://www.hindawi.com
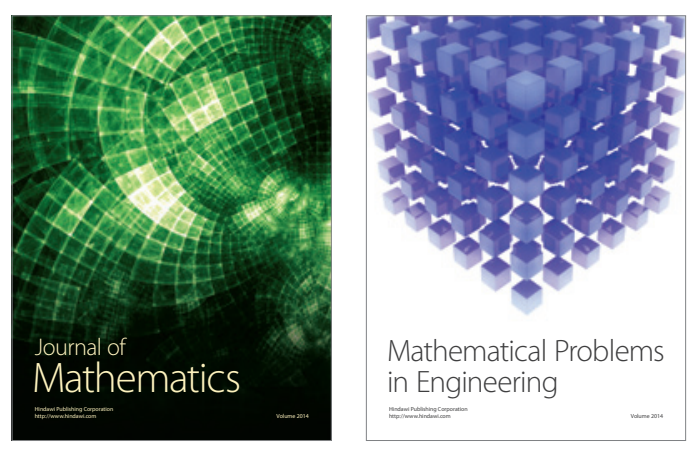

Mathematical Problems in Engineering
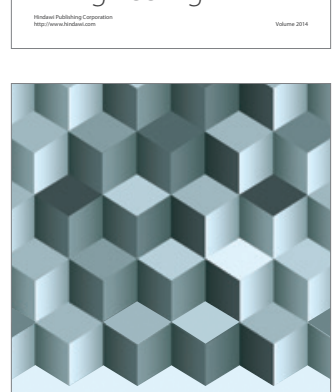

Journal of

Function Spaces
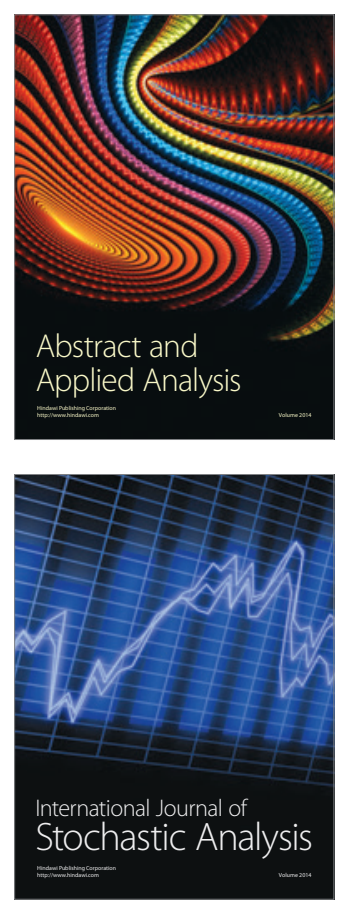

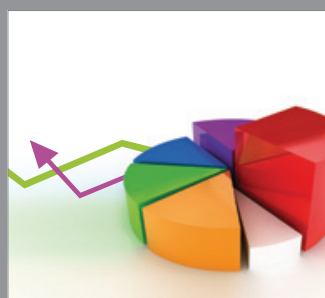

ournal of

Probability and Statistics

Promensencen
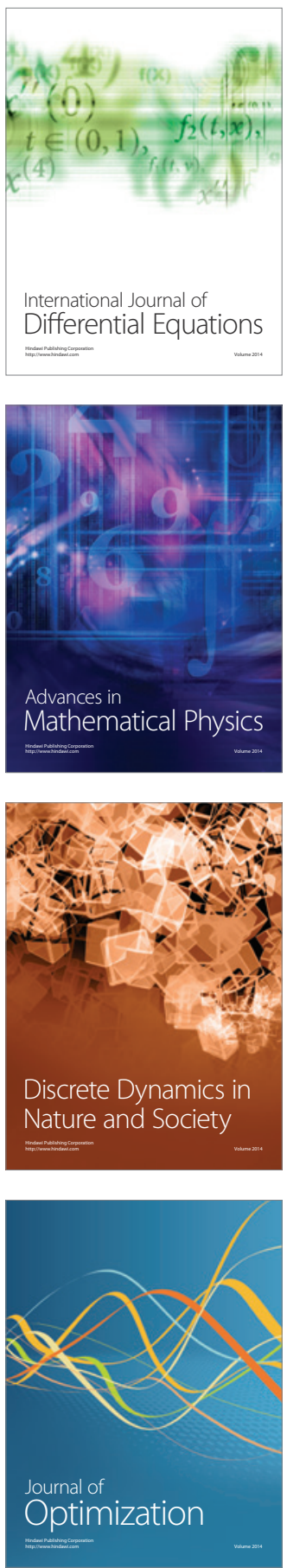